\title{
Chloroquine-induced lipidosis mimicking Fabry disease
}

\author{
Diana Albay ${ }^{1,2}$, Sharon G Adler ${ }^{1,2}$, Jaya Philipose ${ }^{1,2}$, CC Calescibetta ${ }^{2,3}$, \\ Stephen G Romansky ${ }^{4}$ and Arthur H Cohen ${ }^{2,5}$ \\ ${ }^{1}$ Harbor-UCLA Medical Center, Torrance, CA, USA; ${ }^{2} U C L A$ School of Medicine, USA; ${ }^{3}$ St Mary Medical \\ Center, Long Beach, CA, USA; ${ }^{4}$ Memorial Medical Center, Long Beach, CA, USA and ${ }^{5}$ Cedars-Sinai Medical \\ Center, Los Angeles, CA, USA
}

\begin{abstract}
Intracellular accumulation of phospholipids may be a consequence of inherited or acquired metabolic disorders. In Fabry disease, deficiency of $\alpha$-galactosidase A results in storage of globotriasylceramide in numerous cells including endothelium, striated muscle (skeletal, cardiac), smooth muscle, and renal epithelium among others; the ultrastructural appearance of the inclusions is of whorled layers of alternating dense and pale material ('zebra bodies' or myeline figures). Chloroquine therapy may result in storage of biochemically and ultrastructurally similar inclusions in many of the same cells as Fabry disease and often results in similar clinical manifestations. We report a 56-year-old woman with rheumatoid arthritis treated with chloroquine, who developed muscle weakness and renal insufficiency; information regarding therapy was not emphasized at the time of renal biopsy, leading to initial erroneous interpretation of Fabry disease. Following muscle biopsy, genetic and enzyme evaluation, and additional studies on the kidney biopsy, a diagnosis of chloroquine toxicity was established. One year following cessation of chloroquine, renal and muscle dysfunction greatly improved. In chloroquine toxicity, inclusions in glomeruli are not only in visceral epithelial, endothelial and mesangial cells but are in infiltrating monocytes/macrophages, which are most commonly present in the mesangium. Curvilinear bodies, the ultrastructural features of chloroquine toxicity in striated muscle, are not present in renal cells. This report documents differences in appearance, cells affected and morphological differential diagnostic features to distinguish these two entities.
\end{abstract}

Modern Pathology (2005) 18, 733-738, advance online publication, 17 December 2004; doi:10.1038/modpathol.3800344

Keywords: renal lipidoses; chloroquine toxicity; Fabry disease

Tissue damage induced by chloroquine has many morphological similarities to Fabry disease because the cellular inclusions, as defined by their ultrastructural features, are not infrequently considered almost identical. ${ }^{1,2}$ This may result in misdiagnosis, especially when a complete history is not available to the interpreting pathologist or when the pathologist is not aware of therapy. ${ }^{3}$ The inclusions in Fabry disease consist of the stored glycosphingolipid, are characterized as myelin figures or 'zebra bodies', and may be found in renal epithelial cells, endothelial cells, myocardial muscle cells, and in other cells throughout many organs. ${ }^{1,2}$ In contrast, the inclusions that result from chloroquine toxicity are most commonly observed and are clinically

Correspondence: Dr AH Cohen, MD, Department of Pathology, Cedars-Sinai Medical Center, 8700 Beverly Blvd., Room 8738, Los Angeles, CA 90048, USA.

E-mail: cohena@cshs.org

Received 25 May 2004; revised and accepted 18 October 2004; published online 17 December 2004 relevant in renal cells, endothelial cells, and striated muscle cells of both skeletal muscle and myocardium.

In this report, we document the clinical, laboratory, and genetic findings in a woman with rheumatoid arthritis treated with chloroquine, who developed low-grade proteinuria, hematuria, and declining renal function necessitating a renal biopsy. As a result of incomplete historical information, the morphological findings were initially interpreted as Fabry disease. However, subsequent $\alpha$-galactosidase $\mathrm{A}$ activity and genetic mutation analysis did not corroborate this diagnosis. Furthermore, a subsequently performed skeletal muscle biopsy disclosed features of chloroquine toxicity. Retrospective review of the renal morphological findings disclosed subtle differences in the inclusions in this patient compared to those in Fabry disease. In contrast to the previous literature dealing with the structural mimicking of the two diseases, our report includes definitive genetic analysis to exclude a mutation leading to Fabry disease; 
furthermore, we present and emphasize electron microscopic features that allow for distinction of these two entities from one another, especially in renal tissue specimens.

\section{Case report}

A 56-year-old female with severe rheumatoid arthritis was referred by her rheumatologist for nephrological evaluation because of increasing serum creatinine, low-grade proteinuria, hematuria, edema, and progressive muscle weakness. The patient had been treated with celecoxib $400 \mathrm{mg} /$ day and a low dose of chloroquine for 13 months followed by an increase of chloroquine dose to $500 \mathrm{mg}$ /day for 5 months. The usual dose for treatment of rheumatoid arthritis is approximately $400 \mathrm{mg} /$ day initially, followed by maintenance dose of 150-300 mg/day. At the time of consultation, urinalysis showed trace protein, $3+$ occult blood, one large granular cast and a few red blood cells and white blood cells. Renal function was impaired (serum creatinine $(\mathrm{Cr}$ ) $2.2 \mathrm{mg} / \mathrm{dl}$; blood urea nitrogen (BUN) $77 \mathrm{mg} / \mathrm{dl}$; glomerular filtration rate (GFR) $23 \mathrm{ml} / \mathrm{min}$ ). Ultrasound disclosed the kidneys to measure $9.2 \mathrm{~cm} \times$ $3.5 \mathrm{~cm}$ and $10.7 \mathrm{~cm} \times 3.9 \mathrm{~cm}$, with no evidence of hydronephrosis or increased echogenicity. Creatinine phosphokinase and aldolase were within the normal range and electromyographic (EMG) studies revealed nerve conduction velocity changes consistent with both myopathy and axonal neuropathy. Angiokeratomas or rash, acroparethesia, hypohidrosis, or other clinical features of Fabry disease were not present.

Chloroquine and celecoxib were discontinued and she underwent a renal biopsy when $\mathrm{Cr}$ continued to rise despite drug discontinuation. A muscle biopsy was performed several weeks later because of diffuse myopathic features. The clinical impressions included possible steroid myopathy and/or chloroquine toxicity.

\section{Biopsy findings}

\section{Kidney}

Light microscopic examination of routinely prepared paraffin-embedded sections of the kidney biopsy disclosed portions of cortex and medulla with 15 to 18 glomeruli, two of which were completely sclerotic. The remainder appeared similar to one another and were moderately enlarged. Capillary lumina were dilated and filled with cells with vacuolated cytoplasm; the vacuoles were uniform and medium in size (Figure 1). In some, small granular inclusions were identified with periodic acid-methenamine silver stain. Less prominently, the visceral epithelial cells were slightly enlarged and finely and uniformly vacuolated. Capillary walls were single contoured and there was no

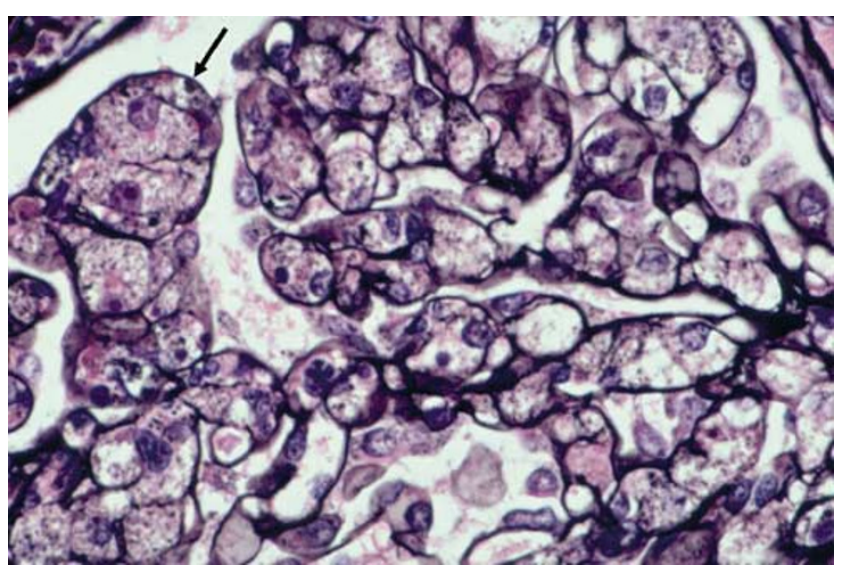

Figure 1 Portion of glomerulus with capillary lumina filled with cells with finely vacuolated cytoplasm, best visualized in lobule indicated with an arrow (periodic acid-methenamine, $\times 400$ ).

increase in cellularity; segments of sclerosis, adhesions, or crescents were absent. There was mild tubular atrophy with interstitial fibrosis. Cells of some proximal and distal tubules were finely vacuolated. Some proximal tubular cells contained protein reabsorption droplets. Arteries and arterioles were unremarkable. There were no medullary alterations.

Seven glomeruli were examined by immunofluorescence microscopy. All were negative for immune reactants. The walls of arterioles stained for C3.

The $1-\mu \mathrm{m}$-thick sections of material that were submitted for electron microscopy, stained with methylene blue-azure II and examined by light microscopy, included two glomeruli, one of which was completely sclerotic. The other displayed dilated capillary lumina filled with cells with vacuolated cytoplasm and few small dense round inclusions. Visceral epithelial cells, on the other hand, were slightly enlarged containing numerous densely stained cytoplasmic granules (Figure 2a). Similar granular structures were in the cytoplasm of some proximal and distal tubular cells. Few dense granules were in the cytoplasm of scattered interstitial cells and few peritubular capillary endothelial cells. Electron microscopic examination indicated that many but not all visceral epithelial cells contained whorled inclusions of lipid. Similar structures were in the cytoplasm of few glomerular capillary and peritubular capillary endothelial cells. Most glomerular capillaries were filled with vacuolated cells, several of which contained small dense round granular inclusions within clear lysosomes. Some of these cells were in the mesangium (Figure 3a). Few endothelial cells in peritubular capillaries, arteries, and arterioles also had similar structures. However, many of these cells also were filled with uniformly dense single membrane-bound inclusions. A mixture of inclusions was also in the cytoplasm of arterial and arteriolar smooth 

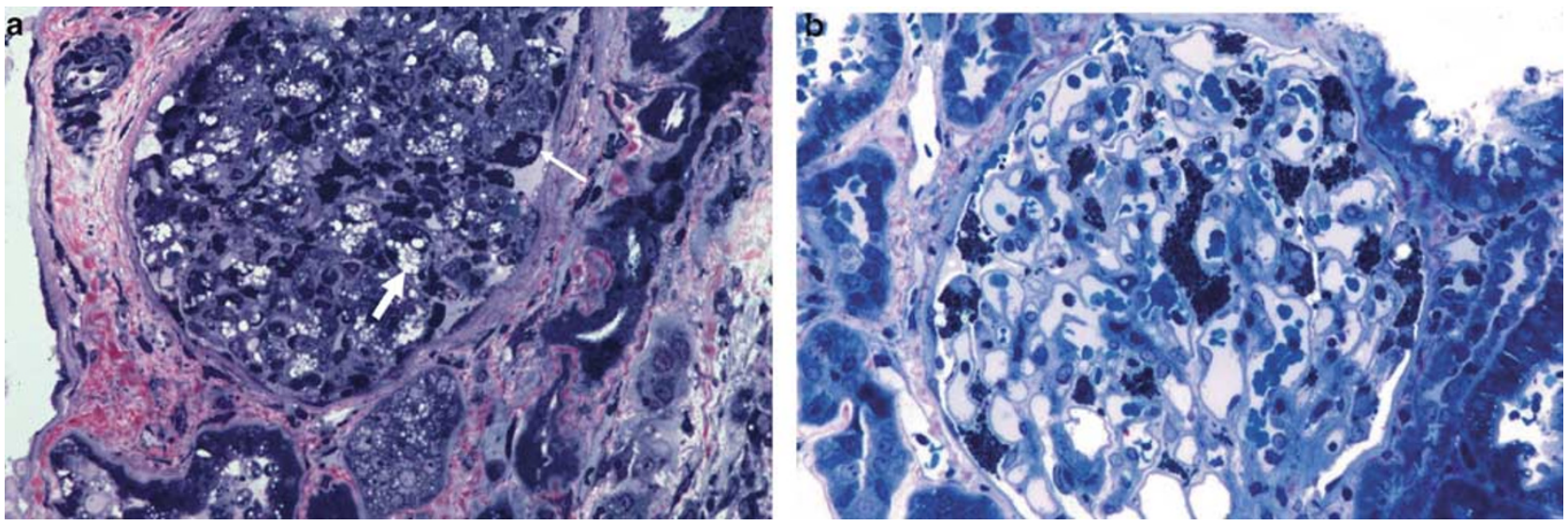

Figure 2 (a) Plastic-embedded section of glomerulus with vacuolated capillary luminal cells (bold arrow) and visceral epithelial cells with dense cytoplasmic granules (thin arrow) (methylene blue-azure II, $\times 200$ ). (b) Glomerulus from a patient with Fabry disease processed in a similar manner. Note the more prominent granularity of visceral epithelial cells and the lack of abnormal capillary luminal cells $(\times 200)$
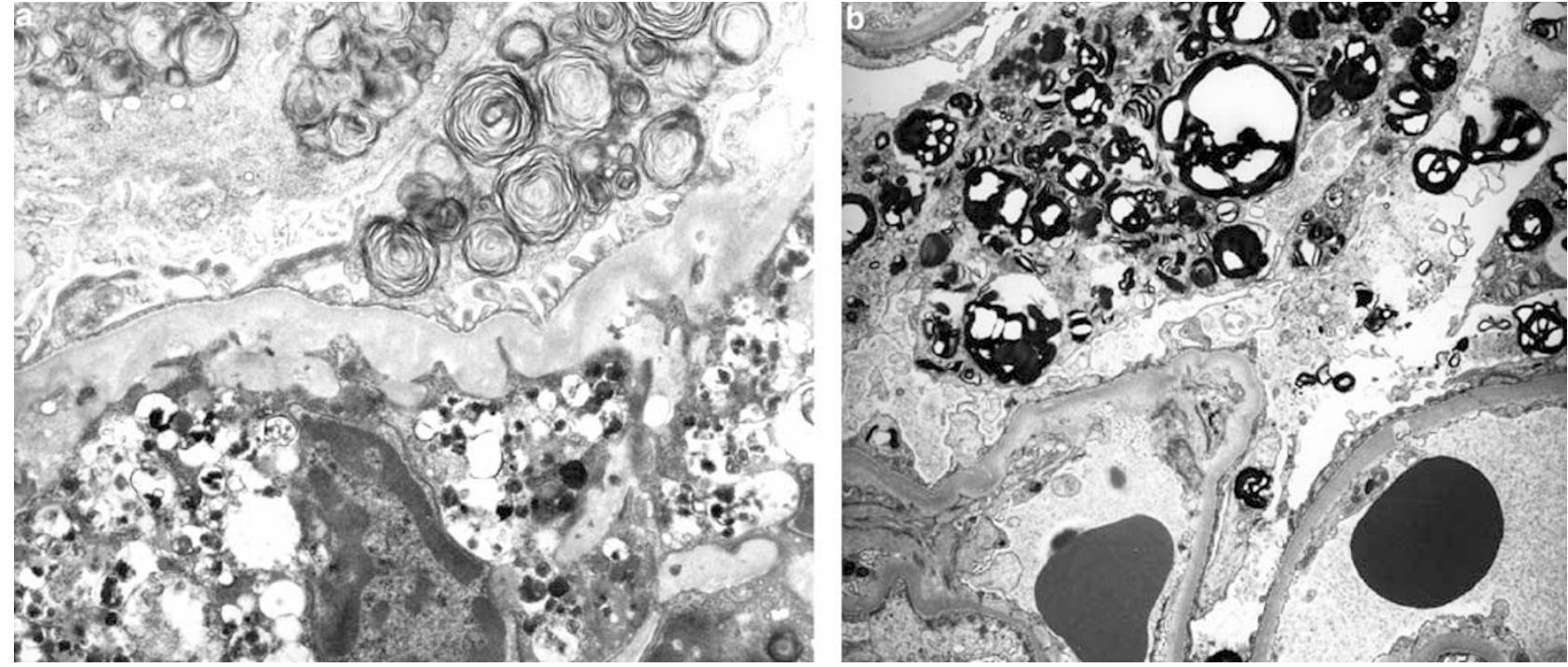

Figure 3 (a) Ultrastructural appearance of a portion of glomerulus. The visceral epithelial cell inclusions are whorled while those in the mesangial cell consist of irregular vacuoles (lysosomes) with dense granules ( $\times 9375)$. (b) Portion of glomerulus from a patient with Fabry disease. The capillary lumina and mesangial regions are devoid of infiltrating cells. The epithelial inclusions are larger and not uniform in size $(\times 8500)$

muscle cells. The foot processes of visceral epithelial cells were partially effaced. There were no electron dense (immune complex) deposits in any location.

For comparison, typical glomerular morphology in a biopsy from a patient with Fabry disease is illustrated as Figure $2 \mathrm{~b}$ to compare with plasticembedded methylene blue-stained $1 \mu \mathrm{m}$-thick sections for electron microscopy. Similarly, Figure $3 \mathrm{~b}$ provides an ultrastructural comparison. See figure legends for description.

Following ultrastructural examination, immunohistochemistry to identify endothelial cells (CD31, CD34) and histiocytes (KP-1) was performed. The vacuolated glomerular capillary luminal cells were positive for KP-1 with a finely granular appearance (Figure 4) and negative for the other antibodies.

\section{Muscle}

Muscle biopsies of the left thigh were snap frozen at $-150^{\circ} \mathrm{C}$ and processed in the usual manner for a battery of histochemical stains including ATPase at preincubations $\mathrm{pH} 9.4,4.4$ and 4.6, NADH-Tr, modified trichome, alkaline phosphatase, phorphorylase A, B, and kinase, cytochrome oxidase, acid phosphatase, osmium, oil red $\mathrm{O}$, nonspecific esterase, PAS, PAS with diastase, and hematoxylin and eosin. One clamped fresh muscle biopsy was fixed 


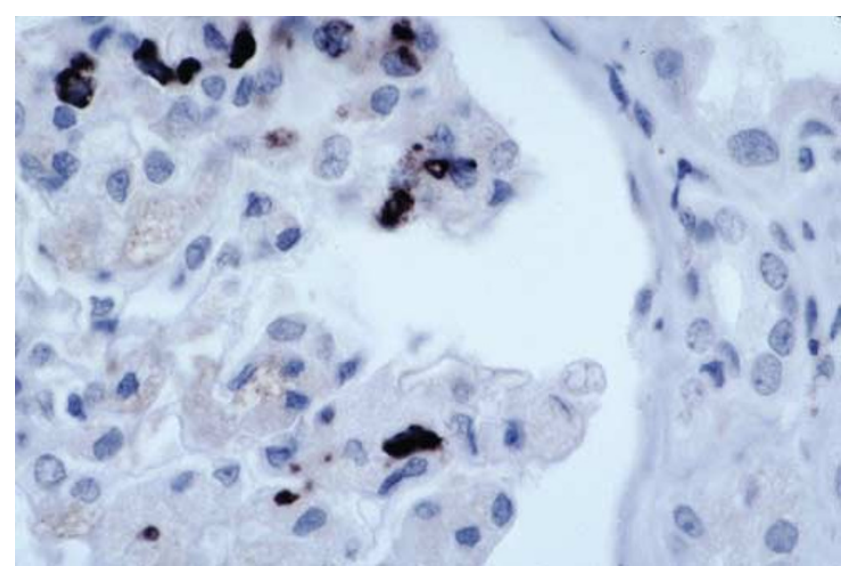

Figure 4 Glomerulus stained with anti KP-1; the cytoplasm of capillary luminal and mesangial cells is positive $(\times 460)$.

in Karnovsky's fixative and subsequently epon embedded, cut at $1 \mu \mathrm{m}$ and stained with methylene blue azure-II and submitted for electron microscopic study.

Light microscopic histochemical findings revealed diffuse myofiber type II atrophy, a few atrophic type I myofibers, and several scattered degenerating and regeneration myofibers. In addition, myofibers containing small, well demarcated, empty cytoplasmic vacuolar inclusions were present.

Electron microscopic study revealed numerous aggregates of curvilinear bodies (CLB) found throughout the cytoplasm of myofibers, distending portions of the sarcomere (Figure 5). CLB were often associated with myelin-like membraneous profiles (myeloid bodies), some mitochondria and occasional lipofuscin granules. CLB were found grouped together in clusters from a few to hundreds of individual curvilinear profiles in a single aggregate.

\section{Follow-up Information}

The patient's plasma $\alpha$-galactosidase A activity level was in the normal range $(33.2 \mathrm{U} / \mathrm{ml}$; range, $8.8-$ $42.6 \mathrm{U} / \mathrm{ml}$ ) and her leukocyte $\alpha$-galactosidase A activity was in the carrier range $(20.5 \mathrm{U} / \mathrm{mg}$; range, 11-182.3 U/mg). In light of these results, mutation analysis was performed. DNA sequencing of all protein coding regions and intron-exon boundaries, performed at Mount Sinai School of Medicine, New York, did not identify a mutation in the patient's $\alpha$ galactosidase A gene. She had no clinical symptoms nor family history of Fabry disease. Thus, in the aggregate, the evidence pointed to an iatrogenic form of Fabry-like disease and not a genetically inherited Fabry disease. At 1 year after discontinuation of chloroquine, the patient's kidney function returned to baseline level (Cr, 1.6; BUN, 26) and hematuria, proteinuria, and edema were

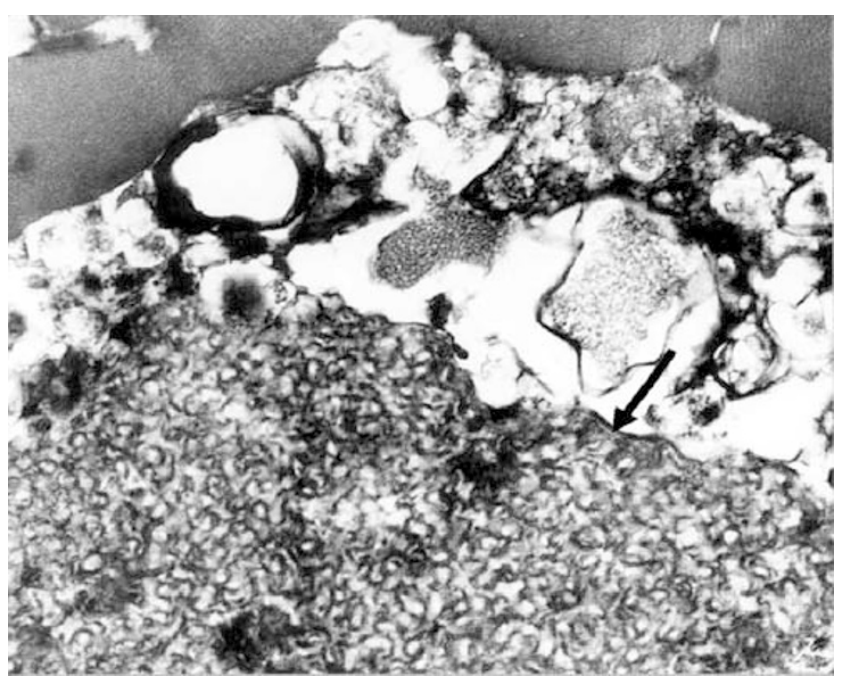

Figure 5 Ultrastructural of skeletal muscle; myriads of curvilinear bodies (arrow) are evident $(\times 10800)$.

resolved. Musculoskeletal complaints also resolved completely.

\section{Discussion}

The morphological abnormalities encountered in this patient's kidney and skeletal muscle biopsies are indicative of cellular injury associated with chloroquine toxicity. Although the inclusions were initially considered by us to represent globotriaosylceramide $\left(\mathrm{Gb}_{3}\right)$, the storage product of Fabry disease, further structural evaluation, normal plasma $\alpha$-galactosidase activity, and lack of mutation in $\alpha$-galactosidase A gene by DNA sequencing dispelled any consideration of a diagnosis of that inherited metabolic disorder. It should be noted again that the duration of treatment with and dosage of chloroquine were not emphasized in the initial history provided with the renal biopsy.

Fabry disease is a rare X-linked lysosomal storage disorder caused by deficient activity of $\alpha$-galactosidase $\mathrm{A}$, resulting in the accumulation of $\mathrm{Gb}_{3}$, also referred to as ceramide trihexoside. ${ }^{4-6}$ As a result of this enzyme deficiency, $\mathrm{Gb}_{3}$ accumulates within many cells resulting in cellular dysfunction and organ system failure. ${ }^{6}$ Primary sites affected by $\mathrm{Gb}_{3}$ accumulation include renal glomerular and tubular epithelial cells, myocardial cells and valvular fibrocytes, neurons of the dorsal root ganglion and autonomic nervous system, and endothelial, perithelial, and smooth muscle cells of the vascular system. ${ }^{5,6}$ Fabry disease has an incidence range of 1 in 40000 to 60000 in males. ${ }^{4}$ Carrier (heterozygous) females also can be affected to a mild or severe degree because of differential X-chromosomal inactivation (Lyonization). ${ }^{4,6,7}$ Major clinical manifestations include cardiomyopathy, coronary artery disease, premature cerebrovascular accidents, 
corneal dystrophy, angiokeratomas, acral paresthesias, and end stage renal disease ${ }^{6}$ Light microscopy of paraffin-embedded tissue reveals vacuolated endothelial, epithelial and muscle lipid-laden cells-foam cells-and electron microscopy reveals dense laminations of lipid material ('zebra bodies') within the lysosomes of affected cells. ${ }^{1,2,6}$ Use of toluidine blue- or methylene blue-stained sections of glutaraldehyde fixed and plastic-embedded sections allows for easy identification of cellular inclusions that appear and are dense granular., ${ }^{1,5}$

Phospholipidosis similar to that observed in Fabry disease has been associated with various drugs; among these, amiodarone ${ }^{8}$ and chloroquine $^{9,10}$ are especially well documented in the literature. Chloroquine, an antimalarial agent also infrequently used in the treatment of various rheumatologic illnesses, has been shown in several animal and human studies to be extensively sequestered in tissues, with the liver, spleen, kidneys, and lungs being the main repositories. The extensive distribution of chloroquine has been attributed to its amphiphilic nature, which allows for lysosomal trapping. ${ }^{11}$ The mechanism by which chloroquine induces lipidosis in tissues remains unknown. In vitro studies in Madin Darby canine kidney cells have shown chloroquine to be a potent inhibitor of lysosomal phospholipase $\mathrm{A}$ and $\mathrm{C}$, suggesting a block in intralysosomal phospholipid catabolism as a possible explanation for the resulting lipidosis. ${ }^{9}$ Inagaki et $a l^{10}$ have not only demonstrated in vitro ultrastructural changes of lysosomal inclusions similar to those observed in Fabry disease but have also shown a specific reduction of $\alpha$-galactosidase A activity associated with chloroquine treatment. Furthermore, this drug's effect on renal function has been documented. In vivo studies in rats demonstrated that chronic treatment was associated with decreased renal function, resulting in a sodium retentive state and edema. ${ }^{12}$

The ultrastructural abnormalities observed in this patient's kidney biopsy, especially involving glomerular capillary lumina, have not been previously emphasized as distinct from the changes of Fabry disease. In chloroquine toxicity as illustrated in our biopsy, the light microscopic features in paraffinembedded sections may include large histiocytes with prominent vacuoles. By electron microscopy, these cells are in capillary lumina or have infiltrated into the mesangium; the inclusions are clear lysosomes with incorporated dense round and variablesized granules. In contrast, endothelial inclusions are round lamellated or solid dense structures. Review of the illustrations in the paper by MüllerHöcker and co-workers ${ }^{3}$ indicates that Figure 2a depicts a capillary luminal cell with ultrastructural features identical to those described in the histiocytes in our biopsy; the authors do not comment on the inclusions other than to note that they are cellular debris. Thus, these electron microscopic abnormalities appear to be unique to severe chlor- oquine cell injury and should serve as an important diagnostic criterion. Macrophage accumulation in capillary lumina in association with other storage diseases has been suggested to contribute to glomerular dysfunction. In single case reports, Santoro et $a l^{13}$ and Newsom et $a l^{14}$ have described patients with glomerular proteinuria and lysosomal storage material in infiltrating macrophages in Gaucher disease $^{13}$ and a poorly characterized glycolipid storage disease to have heavy proteinuria without other explanations.

It is also important to note that ultrastructural findings in muscle biopsies of these patients are also diagnostic of chloroquine toxicity. ${ }^{15}$ Interestingly, the CLB, the diagnostic features in muscle, are not seen in the kidney. Although CLB were previously thought to be a specific ultrastructural marker for ceroid neuronal lipofuscinoses, they are identical morphologically to those associated with chloroquine toxicity.

Until recently, no specific therapy existed for the treatment of Fabry disease and patients were managed with supportive therapy. ${ }^{4}$ Specific enzyme replacement therapy is now available (Fabryzyme ${ }^{\odot}$, Genzyme, Cambridge, MA and Replagal ${ }^{\odot}$, Transkaryotic Therapies, Inc., Cambridge, MA, USA) to supplement supportive therapies. Recently, clinical trials have shown that enzyme replacement with recombinant human $\alpha$-galactosidase A can increase $\alpha$-galactosidase A levels, decrease endothelial inclusions, and improve pain symptoms. ${ }^{16,17}$ However, enzyme replacement therapy costs approximately \$160000 annually. ${ }^{4}$ Thus, with this now readily available but expensive therapy, it is imperative for clinicians to be aware of the rare patient whose Fabry-like syndrome is iatrogenically induced and who does not require $\alpha$-galactosidase A replacement.

\section{References}

1 Cohen AH, Adler SG. Nail-patella syndrome (osteoonychodysplasia), lipodystrophy, Fabry's disease (angiokeratoma corporis diffusum universale), and familial lecithin-cholesterol acyltransferase deficiency. In: Tisher CC, Brenner BM (eds). Renal Pathology with Clinical and Functional Correlations, 2nd edn, JB Lippincott: Philadelphia, PA, 1994, pp 1267-1290.

2 Alroy J, Sabnis S, Kopp JB. Renal pathology in Fabry disease. J Am Soc Nephrol 2002;13:S134-S138.

3 Müller-Höcker J, Schmid H, Weiss M, et al. Chloroquine-induced phospholipidosis of the kidney mimicking Fabry disease: case report and review of the literature. Hum Pathol 2003;34:285-289.

4 Desnick RJ, Brady R, Barranger J, et al. Fabry disease, an under-recognized multisystemic disorder: expert recommendations for diagnosis, management, and enzyme replacement therapy. Ann Intern Med 2003; 138:338-346.

5 Thurberg BL, Rennke H, Colvin RB, et al. Globotriaosycleramide accumulation in the Fabry kidney is 
cleared from multiple cell types after enzyme replacement therapy. Kidney Int 2002;62:1933-1946.

6 Breunig F, Weidemann F, Beer M, et al. Fabry disease: diagnosis and treatment. Kidney Int Suppl 2003;84: S181-S185.

7 Farge D, Nadler S, Wolfe LS, et al. Diagnostic value of kidney biopsy in heterozygous Fabry's disease. Arch Pathol Lab Med 1985;109:85-88.

8 Liu FLW, Cohen RD, Downar E, et al. Amiodarone pulmonary toxicity: functional and ultrastructural evaluation. Thorax 1986;41:100-105.

9 Hostetler KY, Richman DD. Studies on the mechanism of phospholipid storage induced by amantadine and chloroquine in Madin Darby canine kidney cells. Biochem Pharmacol 1982;31:3795-3799.

10 Inagaki M, Katsumoto T, Nanba E, et al. Lysosomal glycosphingolipid storage in chloroquine-induced $\alpha$ galactosidase-deficient human endothelial cells with transformation by simian virus 40: in vitro model of Fabry disease. Acta Neuropathol 1993;85:272-279.

11 Ducharme J, Farinatti R. Clinical pharmacokinetics and metabolism of chloroquine: focus on recent advancements. Clin Pharmacokinet 1996;4: 257-274.

12 Musabayane CT, Ndhlovu CE, Balment RJ. The effects of oral chloroquine administration on kidney function. Renal Failure 1994;16:221-228.

13 Santoro D, Rosenbloom BE, Cohen AH. Gaucher disease with nephrotic syndrome: response to enzyme replacement therapy. Am J Kidney Dis 2002;40:E4.

14 Newsom GD, Stanbaugh GH, Kurtzman NA, et al. Nephrotic syndrome and renal failure associated with a novel glycolipid storage disorder. Am J Nephrol 1988;8:316-321.

15 Neville HE, Maunder-Sewry CA, McDougall J, et al. Chloroquine-induced cytosomes with curvilinear profiles in muscle. Muscle Nerve 1979;2:376-381.

16 Eng CM, Guffon N, Wilcox WR, et al. Safety and efficacy of recombinant human $\alpha$-galactosidase A replacement therapy in Fabry's disease. N Engl J Med 2001;345:9-16.

17 Schiffmann R, Kopp JB, Austin III HA, et al. Enzyme replacement therapy in Fabry disease: a randomized controlled trial. JAMA 2001;285:2743-2749. 